\title{
Progressive Tau Accumulation in Alzheimer Disease: 2-Year Follow-up Study
}

\author{
Hanna Cho ${ }^{1}$, Jae Yong Choi ${ }^{2,3}$, Hye Sun Lee ${ }^{4}$, Jae Hoon Lee ${ }^{2}$, Young Hoon Ryu ${ }^{2}$, Myung Sik Lee ${ }^{1}$, Clifford R. Jack, Jr. ${ }^{5}$, \\ and Chul Hyoung Lyoo ${ }^{1}$ \\ ${ }^{I}$ Department of Neurology, Gangnam Severance Hospital, Yonsei University College of Medicine, Seoul, South Korea; ${ }^{2}$ Department \\ of Nuclear Medicine, Gangnam Severance Hospital, Yonsei University College of Medicine, Seoul, South Korea; ${ }^{3}$ Division of \\ RI-Convergence Research, Korea Institute Radiological and Medical Sciences, Seoul, South Korea; ${ }^{4}$ Biostatistics Collaboration \\ Unit, Gangnam Severance Hospital, Yonsei University College of Medicine, Seoul, South Korea; and ${ }^{5}$ Department of Radiology, \\ Mayo Clinic, Rochester, Minnesota
}

Tau PET enables in vivo visualization and quantitation of tau accumulation in Alzheimer disease (AD). In cross-sectional tau PET studies, tau burden reflects disease severity and phenotypic variation. We investigated longitudinal changes in cortical tau accumulation and their association with cognitive decline in patients with AD. Methods: We enrolled 107 participants (45 amyloid- $\beta$-negative cognitively unimpaired [CU-], 7 amyloid- $\beta$-positive cognitively unimpaired $[\mathrm{CU}+], 31$ with prodromal $A D$ [mild cognitive impairment; $\mathrm{MCl}+]$, and 24 with $A D$ dementia [DEM+]) who completed 2 baseline PET scans $\left({ }^{18} \mathrm{~F}\right.$-flortaucipir and ${ }^{18} \mathrm{~F}$-florbetaben), $\mathrm{MRI}$, and neuropsychologic tests. All participants underwent the same assessments after $2 \mathrm{y}$. After correcting for partial-volume effect, we created SUV ratio (SUVR) images. By using a linear mixed-effect model, we investigated the changes in SUVR across time within each group. We also investigated a correlation between the progression of tau accumulation and cognitive decline. Results: In contrast to no change in global cortical SUVR in the $\mathrm{CU}-$ and $\mathrm{CU}+$ groups during the 2-y period, global cortical SUVR increased by $0.06(2.9 \%)$ in the $\mathrm{MCl}+$ group and $0.19(8.0 \%)$ in the $\mathrm{DEM}+$ group at follow-up. The $\mathrm{MCl}+$ group was associated with additional tau accumulation predominantly in the medial and inferior temporal cortices, whereas the DEM+ group showed increases in the lateral temporal cortex. Progressive tau accumulation occurred in the diffuse cortical areas in the $\mathrm{MCl}+$ patients who developed dementia and the DEM+ patients who showed deterioration of global cognition, whereas there was only a small increase of additional tau accumulation in the lateral temporal cortex in those who did not show worsening of cognition. Deterioration of global cognition and language functions was associated with progression of diffuse tau accumulation in the association neocortex. Conclusion: Progressive tau accumulation occurs in prodromal $A D$ and DEM patients in the cortical areas at different levels of tau accumulation. Progression of cognitive dysfunction may be related to the additional tau accumulation in regions of higher Braak stage. ${ }^{18} \mathrm{~F}$-flortaucipir PET is an imaging biomarker for monitoring the progression of $A D$.

Received Oct. 12, 2018; revision accepted Mar. 18, 2019.

For correspondence or reprints contact either of the following: Chul Hyoung Lyoo, Department of Neurology, Gangnam Severance Hospital, Yonsei University College of Medicine, 20 Eonjuro 63-gil, Gangnam-gu, Seoul, South Korea.

E-mail: lyoochel@yuhs.ac

Young Hoon Ryu, Department of Nuclear Medicine, Gangnam Severance Hospital, Yonsei University College of Medicine, 20 Eonjuro 63-gil, Gangnamgu, Seoul, South Korea.

E-mail: ryuyh@yuhs.ac

Published online Mar. 29, 2019.

COPYRIGHT (C 2019 by the Society of Nuclear Medicine and Molecular Imaging.
Key Words: Alzheimer disease; positron emission tomography; tau; ${ }^{18} \mathrm{~F}$-flortaucipir; longitudinal study

J Nucl Med 2019; 60:1611-1621

DOI: 10.2967/jnumed.118.221697

$\mathbf{P}$

ET imaging with tau-selective radiotracers enables in vivo visualization and quantification of pathologic tau protein in Alzheimer disease (AD) (1). In the last $3 \mathrm{y}$, cross-sectional tau PET studies in $\mathrm{AD}$ have shown distinct binding patterns corresponding to the known topographic distribution pattern of neurofibrillary tangle (NFT) disease and have been shown to reflect clinical and pathologic disease severity and phenotypic variations (2-10). For this reason, tau PET is now considered to be a useful imaging biomarker for diagnosis and disease severity in $\mathrm{AD}$.

Two large longitudinal ${ }^{18} \mathrm{~F}$-flortaucipir PET tau studies from separate groups were recently published $(11,12)$. Patients with cognitive impairment and amyloid- $\beta$ (A $\beta)$ positivity showed an annual increase in cortical SUV ratio (SUVR) by $0.053(3 \%)$ in one study involving 126 participants with a median interval of 1.2 y between scans (11). The other study, in which 142 participants were followed up at 18 mo after baseline, showed an annual increase in SUVR by about 0.05 with a data-driven Parametric Estimation of Reference Signal Intensity (PERSI) reference region in $\mathrm{A} \beta$-positive $\mathrm{AD}$ patients (12).

In this study, we sought to investigate longitudinal changes in cortical ${ }^{18} \mathrm{~F}$-flortaucipir uptake over 2 y in 107 individuals, who included healthy controls and patients with prodromal $\mathrm{AD}$ and $\mathrm{AD}$ dementia (DEM). We also investigated the association between tau propagation and Braak NFT stage and whether the progression of tau accumulation is associated with concomitant cognitive decline.

\section{MATERIALS AND METHODS}

\section{Participants}

We included participants who had completed baseline ${ }^{18} \mathrm{~F}$-flortaucipir PET scans at Gangnam Severance Hospital from January 2015 to July 2016 under the previous tau PET study protocols (Supplemental Table 1; supplemental materials are available at http://jnm.snmjournals.org). At baseline, all participants underwent the neuropsychologic tests, 2 PET scans $\left({ }^{18} \mathrm{~F}\right.$-flortaucipir for tau and ${ }^{18} \mathrm{~F}$-florbetaben for $\left.\mathrm{A} \beta\right)$, and 
MR imaging studies. On the basis of cognitive status, neuropsychologic test performance, and $\mathrm{A} \beta$ positivity determined by agreement between 2 nuclear medicine specialists using a validated visual assessment method for ${ }^{18} \mathrm{~F}$-florbetaben PET $(13,14)$, we selected the DEM patients who fulfilled the diagnostic criteria for "probable DEM with evidence of the AD pathophysiologic process" (15) and mild cognitive impairment (MCI) patients who fulfilled the criteria for "MCI due to AD with intermediate or high likelihood" proposed by the National Institute on Aging-Alzheimer Association (16). A second diagnosis at follow-up was confirmed using the same diagnostic criteria. All AD patients initially presented with memory impairment without showing atypical features suggesting posterior cortical atrophy, logopenic aphasia, or frontal-variant AD. We also included healthy controls who were "cognitively unimpaired" (CU) by showing normal performance on baseline neuropsychologic tests and no abnormalities on brain MRI. Finally, 107 participants were included in this study, and we classified the participants into 4 diagnostic groups (45 $\mathrm{A} \beta$-negative $\mathrm{CU}[\mathrm{CU}-]$, $7 \mathrm{~A} \beta$-positive $\mathrm{CU}[\mathrm{CU}+], 31 \mathrm{~A} \beta$-positive $\mathrm{MCI}[\mathrm{MCI}+]$, and $24 \mathrm{~A} \beta$-positive $\mathrm{DEM}[\mathrm{DEM}+])$.

At follow-up, all participants underwent the same neuropsychologic tests, PET scans, and MRI studies as for the baseline assessments. Apolipoprotein E (ApoE) genotypes were determined at baseline. Follow-up assessments were performed within 3 mo from the presumed date for the 2-y follow-up.

This study was approved by the institutional review board of Gangnam Severance Hospital, and written informed consent was obtained from all subjects.

\section{Neuropsychologic Tests}

Using the Seoul Neuropsychological Screening Battery, cognitive function was evaluated at both baseline and follow-up (17). The battery includes scorable items for global cognitive function and 6 cognitive domains: the Korean version of the Mini-Mental State Examination (MMSE) and Clinical Dementia Rating Sum of Boxes, the Digit Span Backward test (attention), the Boston Naming Test (language), the Rey-Osterrieth Complex Figure Test (visuospatial function), the Seoul Verbal Learning Test-Delayed Recall (verbal memory), the Rey-Osterrieth Complex Figure Test-Delayed Recall (visual memory), and the Controlled Oral Word Association TestSemantics (frontal/executive function).

\section{Acquisition of PET and MR Images}

All participants underwent ${ }^{18} \mathrm{~F}$-flortaucipir and ${ }^{18} \mathrm{~F}$-florbetaben PET scans on separate days. At $80 \mathrm{~min}\left({ }^{18} \mathrm{~F}\right.$-flortaucipir) or $90 \mathrm{~min}\left({ }^{18} \mathrm{~F}-\right.$ florbetaben) after the injection of radiotracers, emission scans were acquired for 20 min with a Biograph mCT PET/CT scanner (Siemens Medical Solutions) after applying a head holder to minimize head motion. Before the PET scan, brain CT images were acquired for later attenuation correction. Finally, PET images were reconstructed in a $256 \times 256 \times 223$ matrix with a $1.591 \times 1.591 \times 1 \mathrm{~mm}$ voxel size using the ordered-subsets expectation maximization algorithm after attenuation and scatter correction.

T1-weighted MR images were also acquired with 3-dimensional spoiled gradient-recalled sequences (repetition time, $8.28 \mathrm{~ms}$; echo time, 1.6-11.0 ms; flip angle, $20^{\circ}$; $512 \times 512$ matrix; voxel spacing, $0.43 \times 0.43 \times 1 \mathrm{~mm}$ ) in a 3.0-T MR scanner (Discovery MR750; GE Healthcare).

\section{Image Processing Steps}

We used FreeSurfer (version 5.3; Massachusetts General Hospital, Harvard Medical School; http://surfer.nmr.mgh.harvard.edu) software for creating participant-specific volumes of interest (VOIs), extracting surface structures, and measuring cortical thickness as described in a previous publication (2). By using the parcellated segments, we created participant-specific composite VOI mask images including 16 cortical and 5 subcortical regions after merging anatomically related regions. Regional volumes were measured by counting the voxels within each region.

Statistical parametric mapping (version 12; Wellcome Trust Centre for Neuroimaging) and in-house software implemented in MATLAB (version R2015b; The MathWorks) were used to process the PET images and measure regional uptake. PET images were coregistered to individual MR images. By using the masks for parcellated regions, the partial-volume effect (PVE) was corrected with the region-based voxelwise method (18). For creating SUVR images, we primarily used the cerebellar crus median obtained by overlaying the template VOI for the cerebellar crus on the spatially normalized PET images (11), and we additionally used the PERSI reference region for ${ }^{18} \mathrm{~F}$-flortaucipir PET studies (12). Finally, by overlaying the participant-specific composite VOI masks, we measured regional SUVR. We also measured ${ }^{18} \mathrm{~F}$-flortaucipir SUVR for the composite regions corresponding to each Braak stage by volume-weighted averaging of VOI values. The hippocampus was excluded because of known off-target binding of ${ }^{18} \mathrm{~F}$-flortaucipir. We focused primarily on the PVE-corrected data for analysis but also analyzed the PVE-uncorrected data.

To obtain the participant-specific PERSI reference region, we selected white matter voxels with an intensity within the full width at half maximum of an imaginary nonspecific intensity curve obtained by fitting the white matter intensity histogram into the binominal gaussian distribution (12). The same PERSI reference VOI mask was used for creating both PVE-uncorrected and PVE-corrected ${ }^{18} \mathrm{~F}-$-flortaucipir SUVR images.

Cortical uptake was also mapped on the white matter surface by overlaying the values of voxels corresponding to the midpoint between a vertex on the white matter surface and the corresponding vertex on the gray matter surface. As with the volume-based correction for PVE, we created first PVE-corrected surface maps and then surface SUVR maps. Surface-based SUVR and cortical thickness maps were spatially normalized to a template surface and smoothed on the 2-dimensional surface by a gaussian kernel of $8 \mathrm{~mm}$ in full width at half maximum.

\section{Statistical Analysis}

SPSS (version 23; IBM Corp.) was used for statistical analysis of demographic data. The $\chi^{2}$ test and a correction for multiple comparisons with the Bonferroni method were used to compare categoric variables. For the comparisons of continuous demographic data and cognitive test performance, we used an ANOVA model with a Bonferroni post hoc test. Performance on cognitive function tests was compared between groups using a general linear model with age, sex, years of education, and presence of ApoE $\varepsilon 4$ as covariates and the Bonferroni method for correcting multiple comparisons.

We primarily analyzed longitudinal changes in cognitive test performance, regional SUVR, and VOI in each group using the linear mixed-effect model implemented in MATLAB with baseline age, sex, years of education, presence of ApoE $\varepsilon 4$ allele, and time interval between baseline and follow-up as fixed factors and subject as a random factor under the assumption that the intercepts can differ between subjects. Likewise, longitudinal changes in the SUVR maps on the surface were analyzed with the same model in MATLAB. For both VOI- and surface-based analysis, we chose a random-intercept model rather than a random-intercept-and-slope model to reduce computational burden and later confirmed no difference between the results driven by the 2 models.

For the correlation analysis assessing the change in SUVR and cognitive function, we used a multiple-regression model with regional changes in SUVR over the 2-y follow-up as an independent variable and cognitive function as a dependent variable and with age, sex, years of education, and presence of ApoE $\varepsilon 4$ allele as covariates. 
For both VOI- and surface-based analysis, multiple comparisons were corrected using the Benjamini-Hochberg false-discovery rate method (19).

\section{RESULTS}

\section{Demographic Characteristics and Neuropsychologic Tests}

The demographic data and neuropsychologic tests are summarized in Table 1. The MCI + and DEM+ groups were older than the $\mathrm{CU}-$ group. The proportion of women was higher and disease duration longer in the DEM+ group than in the MCI+ group. The frequency of ApoE $\varepsilon 4$ carriers was higher in the MCI+ and $\mathrm{DEM}+$ groups than in the $\mathrm{CU}-$ group. Years of education did not differ among the groups. All participants completed the baseline and follow-up assessments, with an average interval of $24.0 \pm 1.5 \mathrm{mo}$. Mean intervals were slightly shorter for the MCI+ and DEM+ groups (23.7 and $23.1 \mathrm{mo}$, respectively) than for the CU- group (24.7 mo).

During the 2-y follow-up, 10 of the $45 \mathrm{CU}-$ individuals and 3 of the $7 \mathrm{CU}+$ individuals progressed to MCI and 20 of the 31 MCI+ individuals progressed to clinically overt dementia. All 24 $\mathrm{DEM}+$ individuals remained in the dementia state at follow-up. Meanwhile, $5 \mathrm{MCI}+$ individuals reverted to normal cognition. Thirteen DEM + individuals showed a worsening of global cognition (decrease in MMSE score $\geq 3$ ), whereas the other $11 \mathrm{DEM}+$ individuals did not progress (decrease in MMSE score $<3$ ).

At both baseline and follow-up, the DEM+ group performed significantly worse on all neuropsychologic tests than the CUgroup and significantly worse on all tests other than the attention function test than the $\mathrm{CU}+$ group. When compared with the $\mathrm{MCI}+$ group, the DEM+ group showed worse performance in global cognition, visuospatial function, and frontal/executive function at baseline and follow-up and in attention and language function at follow-up. With the exception of the attention and visuospatial function tests at both baseline and follow-up, the $\mathrm{MCI}+$ group showed worse performance than the CU- group. When compared with the $\mathrm{CU}+$ group, the MCI+ group performed worse on memory function at baseline and follow-up. Three groups (CU-, MCI+, and DEM+) showed a significant deterioration in global cognitive function as determined by MMSE $(P<0.001)$ and Clinical Dementia Rating Sum of Boxes scores (CU-, $P=0.005$; MCI,$+ P<0.001$; DEM + , $P=0.001)$ during the interval. The $\mathrm{CU}-$ group showed a significant deterioration in verbal memory $(P<0.001)$ and visuospatial function $(P<0.001)$; the MCI + group, in language $(P=0.009)$ and visual memory function $(P=0.007)$; and the DEM+ group, in language function $(P<0.001)$.

\section{Longitudinal Changes in ${ }^{18} \mathrm{~F}$-Flortaucipir Uptake}

Some examples of the baseline and follow-up ${ }^{18} \mathrm{~F}$-flortaucipir PET images are shown in Figure 1A. ${ }^{18} \mathrm{~F}$-flortaucipir uptake tended to increase in and around the cortical regions with intense uptake at baseline in the MCI+ and DEM+ groups on visual inspection of individual PET images. Conversely, the CU - group did not show such an increase at follow-up, although there was a small increase in the medial temporal regions in some CUparticipants.

During the 2-y follow-up, the global cortical ${ }^{18} \mathrm{~F}$-flortaucipir SUVR of the MCI+ and DEM+ groups increased by 0.06 $(2.9 \%)$ and $0.19(8.0 \%)$, respectively. Those groups showed an increase in uptake most prominently in the medial and lateral temporal regions corresponding to Braak NFT stages I-IV $(\triangle \mathrm{SUVR}$ in the composite stage I-IV region: $\mathrm{MCI}+, 0.14$ [6.7\%]; DEM,$+ 0.29[11.0 \%])$, and the increase was attenuated in regions of higher Braak stage. Interestingly, stage I-II regions showed a maximal increase in SUVR (0.27 [9.3\%]) in the MCI+ group, whereas the increase in SUVR in stage III-IV regions $(0.29$ [11.2\%]) was maximal in the DEM+ group. Although global cortical SUVR barely changed in the CU- $(-0.01[-0.7 \%])$ and $\mathrm{CU}+(-0.01[-0.3 \%])$ groups, the maximal increase was found in stage I-II regions (CU-, 0.03 [1.8\%]; CU+, 0.13 [6.6\%]) (Fig. 1B; Table 2).

When not corrected for PVE, the changes in SUVR were almost half those obtained after correcting for PVE. The global cortical SUVR of the MCI+ and DEM+ groups increased at follow-up by only $0.03(1.5 \%)$ and $0.08(5.0 \%)$, respectively, and the increase in SUVR in the composite stage I-IV region was 0.07 (4.0\%) in the $\mathrm{MCI}+$ group and $0.13(6.7 \%)$ in the DEM+ group (Table 2).

In VOI-based statistical analyses of smaller regions, a significant increase in ${ }^{18} \mathrm{~F}$-flortaucipir SUVR was found in the lateral temporal, entorhinal, parahippocampal, posterior cingulate, and insula cortices and the amygdala in the MCI+ group (most prominently in the inferior temporal and parahippocampal cortices) and in diffuse cortical areas except for the sensorimotor, superior parietal, and cingulate cortices in the DEM+ group (most prominently in the middle temporal cortex). Very small increases in SUVR in the medial temporal regions were observed in both the $\mathrm{CU}-$ group and the CU+ group, but these differences did not reach statistical significance (Fig. 2). In $62 \mathrm{~A} \beta$-positive participants, there was a positive correlation between baseline SUVR and the change in SUVR in all cortical regions except the sensorimotor, superior parietal, precuneal, and posterior cingulate cortices and the amygdala $(P<0.05)$. No cortical region showed a negative correlation. Although baseline global cortical SUVs negatively correlated with baseline age in these $62 \mathrm{~A} \beta$-positive participants $(P=0.003)$, there was only a weak trend toward a negative correlation between baseline age and change in global cortical SUVR $(P=0.087)$, and only the change in prefrontal SUVR showed a weak negative correlation $(P=$ 0.030 ), which did not survive correction for multiple comparisons. Additionally, in $45 \mathrm{CU}-$ individuals, only the change in SUVR in the entorhinal cortex showed a weak positive correlation with baseline age $(P=0.015)$, but the correlation did not survive correction for multiple comparisons.

Similar to the VOI-based analysis, surface-based analysis revealed an increase in cortical ${ }^{18} \mathrm{~F}$-flortaucipir uptake during the 2-y follow-up. The increase was greatest in the DEM+ group, followed by the MCI+ group. These groups showed a prominent increase in the medial, basal, and lateral temporal regions. Interestingly, the DEM+ group showed a less significant increase in the medial and basal temporal regions than the MCI+ group. Even the CU- group showed a small increase in uptake in the medial and basal temporal regions, although the increase was not significant (Fig. 2).

PVE-uncorrected data showed patterns of increase similar to those found for PVE-corrected data. However, the increase in the PVE-uncorrected SUVR in the DEM+ group was much smaller than the increase in the PVE-corrected SUVR (Supplemental Fig. 1).

Eleven MCI+ patients who did not progress to clinical dementia showed an increase in SUVR in the inferior temporal cortex, a difference that did not survive correction for multiple 
TABLE 1

Baseline Demographic Characteristics and Changes in Cognitive Function

\begin{tabular}{|c|c|c|c|c|}
\hline Characteristic & CU- & $\mathrm{CU}+$ & $\mathrm{MCl}+$ & DEM+ \\
\hline$n$ & 45 & 7 & 31 & 24 \\
\hline Baseline age (y) & $66.8 \pm 9.7$ & $71.7 \pm 4.2$ & $73.2 \pm 7.3^{*}$ & $73.3 \pm 8.9^{*}$ \\
\hline \multicolumn{5}{|l|}{$\operatorname{Sex}(n)$} \\
\hline$M$ & 18 & 3 & 18 & 4 \\
\hline $\mathrm{F}$ & 27 & 4 & 13 & $20^{\dagger}$ \\
\hline Education (y) & $12.2 \pm 5.0$ & $13.0 \pm 4.1$ & $11.9 \pm 4.9$ & $9.5 \pm 5.7$ \\
\hline Duration (y) & NA & NA & $2.8 \pm 1.4$ & $3.8 \pm 1.3^{\dagger}$ \\
\hline \multicolumn{5}{|l|}{$\operatorname{ApoE}(n)$} \\
\hline$\varepsilon 4+$ & $8(18 \%)$ & 2 (29\%) & $16(52 \%)$ & 17 (71\%) \\
\hline$\varepsilon 4-$ & 37 & 5 & 15 & 7 \\
\hline Interval ${ }^{\ddagger}$ (mo) & $24.7 \pm 1.4$ & $24.4 \pm 1.9$ & $23.7 \pm 1.1^{*}$ & $23.1 \pm 1.4^{*}$ \\
\hline Baseline centiloid & $9.1 \pm 11.2$ & $81.4 \pm 46.4^{\star}$ & $79.7 \pm 40.8^{\star}$ & $110.6 \pm 36.7^{\star \star}$ \\
\hline \multicolumn{5}{|l|}{ MMSE } \\
\hline Baseline & $28.2 \pm 1.8$ & $28.1 \pm 2.1$ & $25.2 \pm 3.2^{*}$ & $19.1 \pm 4.5^{\star+十}$ \\
\hline Follow-up & $27.1 \pm 3.3^{\S}$ & $27.3 \pm 2.9$ & $22.2 \pm 5.0^{\star \star \mid \S}$ & $15.7 \pm 5.2^{\star \star \uparrow \rrbracket}$ \\
\hline \multicolumn{5}{|l|}{ CDR-SB } \\
\hline Baseline & $0.0 \pm 0.0$ & $0.0 \pm 0.0$ & $1.7 \pm 0.9 * \pi$ & $4.8 \pm 1.8^{\star+1}$ \\
\hline Follow-up & $1.3 \pm 3.0^{\S}$ & $0.7 \pm 1.1$ & $3.1 \pm 1.9^{\star \S}$ & $7.0 \pm 3.0^{\star *+ศ \S}$ \\
\hline \multicolumn{5}{|l|}{ DS-BW } \\
\hline Baseline & $4.1 \pm 1.6$ & $3.3 \pm 0.8$ & $3.7 \pm 1.5$ & $2.8 \pm 1.3$ \\
\hline Follow-up & $4.2 \pm 1.3$ & $3.4 \pm 0.5$ & $3.8 \pm 1.4$ & $2.4 \pm 1.5^{\star \dagger}$ \\
\hline \multicolumn{5}{|l|}{ BNT } \\
\hline Baseline & $50.2 \pm 7.7$ & $48.7 \pm 3.0$ & $42.1 \pm 8.2^{\star}$ & $34.3 \pm 11.5^{\star \star ा}$ \\
\hline Follow-up & $49.2 \pm 8.3$ & $48.3 \pm 5.7$ & $39.5 \pm 9.4^{\star \S}$ & $26.2 \pm 10.2^{\star \mp \rrbracket \mid ~}$ \\
\hline \multicolumn{5}{|l|}{ RCFT } \\
\hline Baseline & $32.9 \pm 4.7$ & $34.0 \pm 2.0$ & $30.4 \pm 6.8$ & $22.7 \pm 10.7^{\star \star 丿 ⿻ ~}$ \\
\hline Follow-up & $31.4 \pm 5.7^{\S}$ & $33.0 \pm 2.7$ & $28.6 \pm 7.5$ & $18.8 \pm 11.3^{\star+1}$ \\
\hline \multicolumn{5}{|l|}{ SVLT-DR } \\
\hline Baseline & $6.6 \pm 2.5$ & $6.0 \pm 2.2$ & $1.4 \pm 2.2^{\star \pi}$ & $0.8 \pm 2.1^{\star \star ा}$ \\
\hline Follow-up & $5.5 \pm 2.4^{\S}$ & $4.6 \pm 3.2$ & $1.5 \pm 2.4^{\star \star ⿻ ~}$ & $0.3 \pm 1.1^{\star \pi}$ \\
\hline \multicolumn{5}{|l|}{ RCFT-DR } \\
\hline Baseline & $16.0 \pm 7.3$ & $15.5 \pm 5.3$ & $6.5 \pm 5.7^{\star \bullet}$ & $2.1 \pm 3.7^{\star \star ा}$ \\
\hline Follow-up & $16.0 \pm 8.4$ & $15.5 \pm 6.9$ & $4.2 \pm 6.1^{\star \star \Phi \S ~}$ & $0.7 \pm 1.9 * \pi$ \\
\hline \multicolumn{5}{|l|}{ COWAT } \\
\hline Baseline & $16.2 \pm 4.4$ & $15.3 \pm 6.1$ & $13.1 \pm 4.9$ & $8.6 \pm 4.1^{\star \star 十 ⿻ ा ~}$ \\
\hline Follow-up & $15.7 \pm 4.2$ & $14.7 \pm 6.4$ & $12.4 \pm 5.3^{*}$ & $7.0 \pm 4.0^{\star}+\llbracket$ \\
\hline \multicolumn{5}{|c|}{$\begin{array}{l}{ }^{\star} P<0.05 \text { for comparisons between } \mathrm{CU}-\text { and each group. } \\
{ }^{\dagger} P<0.05 \text { for comparisons between } \mathrm{MCI}+\text { and DEM }+ \text { groups. } \\
{ }^{\ddagger} \text { From baseline to follow-up. } \\
{ }^{\mathrm{I} P}<0.05 \text { for comparisons between } \mathrm{CU}+\text { and } \mathrm{MCl} / \mathrm{DEM}+\text { groups. } \\
{ }^{\S} P<0.05 \text { for significant changes between baseline and follow-up. } \\
\text { CDR-SB = Clinical Dementia Rating Sum of Boxes; DS-BW = Digit Span Backward; BNT = Boston Naming Test; RCFT = Rey- } \\
\text { Osterrieth Complex Figure Test; SVLT-DR = Seoul Verbal Learning Test-Delayed Recall; RCFT-DR = Rey-Osterrieth Complex Figure } \\
\text { Test-Delayed Recall; COWAT = Controlled Oral Word Association Test-Semantics; NA = not applicable or not available. } \\
\text { Qualitative data are expressed as numbers; continuous data are expressed as mean } \pm \text { SD. }\end{array}$} \\
\hline
\end{tabular}

1614 The Journal of Nuclear Medicine • Vol. 60 • No. 11 • November 2019 


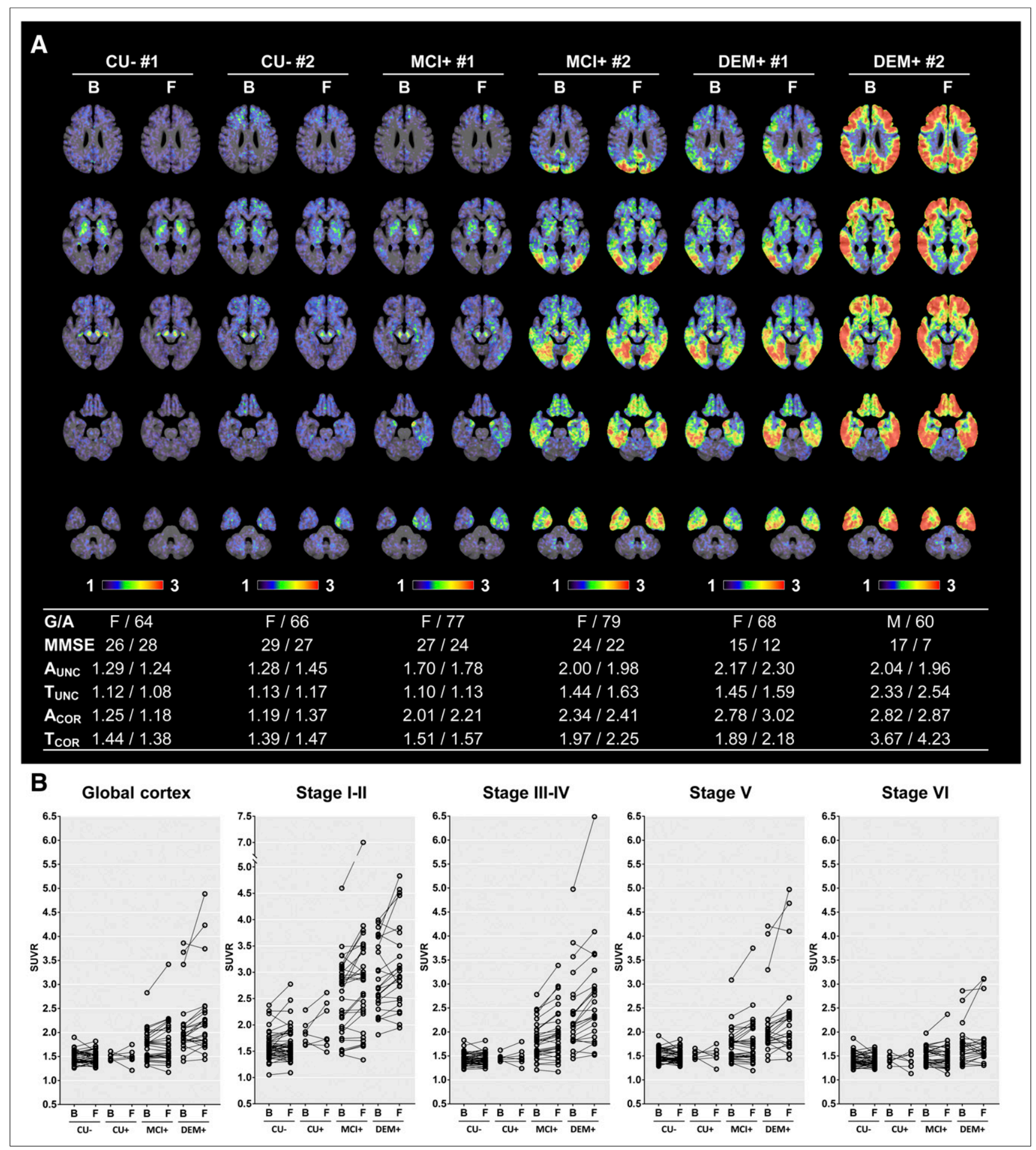

FIGURE 1. Examples of ${ }^{18} \mathrm{~F}$-flortaucipir PET SUVR images created with cerebellar crus as reference region and their longitudinal changes in PVEcorrected SUVR obtained at baseline and follow-up. (A) Examples of spatially normalized PET images uncorrected for PVE, with ${ }^{18} \mathrm{~F}$-florbetaben and ${ }^{18} \mathrm{~F}$-flortaucipir SUVR and MMSE scores presented below the images. (B) ${ }^{18} \mathrm{~F}$-flortaucipir SUVR measured in global cortical gray matter and composite regions corresponding to different Braak stages. $B=$ baseline; $F=$ follow-up; $G / A=$ sex/age; $A$ and $T=$ global cortical SUVR of ${ }^{18} \mathrm{~F}$-florbetaben or ${ }^{18} \mathrm{~F}$-flortaucipir PET, respectively; COR and UNC = SUVR with and without PVE correction, respectively.

comparisons. Meanwhile, $20 \mathrm{MCI}$ progressors showed an increase in SUVR in all cortical regions except the sensorimotor cortex, most prominently in the inferior and middle temporal cortices and medial temporal regions (Fig. 3A). Eleven DEM+ patients who did not show a progression of global cognitive function exhibited an increase in SUVR in the middle temporal cortex, a difference that did not survive correction for multiple comparisons. The other $13 \mathrm{DEM}+$ patients whose global 
TABLE 2

Longitudinal Changes in Cortical ${ }^{18} \mathrm{~F}$-Flortaucipir SUVR Obtained with Cerebellar Crus as Reference Region

\begin{tabular}{|c|c|c|c|c|}
\hline Parameter & CU- & $\mathrm{CU}+$ & $\mathrm{MCl}+$ & DEM+ \\
\hline \multicolumn{5}{|l|}{ PVE-corrected } \\
\hline \multicolumn{5}{|l|}{ cGM } \\
\hline$\Delta$ SUVR & $-0.01 \pm 0.10$ & $-0.01 \pm 0.16$ & $0.06 \pm 0.15$ & $0.19 \pm 0.34$ \\
\hline$\%$ & $-0.7 \pm 6.6$ & $-0.3 \pm 10.6$ & $2.9 \pm 7.4$ & $8.0 \pm 11.9$ \\
\hline \multicolumn{5}{|l|}{ Stage I-II } \\
\hline$\Delta$ SUVR & $0.03 \pm 0.15$ & $0.13 \pm 0.27$ & $0.27 \pm 0.49$ & $0.21 \pm 0.39$ \\
\hline$\%$ & $1.8 \pm 8.9$ & $6.6 \pm 14.2$ & $9.3 \pm 13.4$ & $6.9 \pm 11.3$ \\
\hline \multicolumn{5}{|l|}{ Stage III-IV } \\
\hline$\Delta$ SUVR & $0.00 \pm 0.08$ & $0.02 \pm 0.12$ & $0.14 \pm 0.18$ & $0.29 \pm 0.34$ \\
\hline$\%$ & $0.0 \pm 5.0$ & $1.2 \pm 7.9$ & $6.8 \pm 7.5$ & $11.2 \pm 10.0$ \\
\hline \multicolumn{5}{|l|}{ Stage V } \\
\hline$\Delta$ SUVR & $-0.02 \pm 0.11$ & $-0.01 \pm 0.17$ & $0.06 \pm 0.17$ & $0.20 \pm 0.38$ \\
\hline$\%$ & $-0.9 \pm 7.2$ & $-0.5 \pm 11.4$ & $2.5 \pm 8.2$ & $8.2 \pm 13.6$ \\
\hline \multicolumn{5}{|l|}{ Stage VI } \\
\hline$\Delta$ SUVR & $-0.03 \pm 0.12$ & $-0.03 \pm 0.18$ & $0.00 \pm 0.13$ & $0.07 \pm 0.24$ \\
\hline$\%$ & $-1.4 \pm 8.0$ & $-1.7 \pm 12.3$ & $-0.5 \pm 7.7$ & $3.4 \pm 11.7$ \\
\hline \multicolumn{5}{|c|}{ PVE-uncorrected } \\
\hline \multicolumn{5}{|c|}{ cGM } \\
\hline$\Delta$ SUVR & $-0.02 \pm 0.08$ & $-0.01 \pm 0.12$ & $0.03 \pm 0.09$ & $0.08 \pm 0.19$ \\
\hline$\%$ & $-1.2 \pm 6.5$ & $-0.7 \pm 10.9$ & $1.5 \pm 6.4$ & $5.0 \pm 10.1$ \\
\hline \multicolumn{5}{|l|}{ Stage I-II } \\
\hline$\Delta$ SUVR & $0.00 \pm 0.09$ & $0.04 \pm 0.17$ & $0.04 \pm 0.11$ & $0.03 \pm 0.21$ \\
\hline$\%$ & $0.1 \pm 6.4$ & $2.1 \pm 12.8$ & $2.4 \pm 6.5$ & $1.4 \pm 10.0$ \\
\hline \multicolumn{5}{|l|}{ Stage III-IV } \\
\hline$\Delta$ SUVR & $-0.01 \pm 0.06$ & $0.01 \pm 0.11$ & $0.07 \pm 0.10$ & $0.13 \pm 0.19$ \\
\hline$\%$ & $-0.6 \pm 5.1$ & $0.3 \pm 9.3$ & $4.1 \pm 5.7$ & $6.9 \pm 8.4$ \\
\hline \multicolumn{5}{|l|}{ Stage V } \\
\hline$\Delta S U V R$ & $-0.02 \pm 0.09$ & $-0.01 \pm 0.13$ & $0.02 \pm 0.10$ & $0.09 \pm 0.22$ \\
\hline$\%$ & $-1.3 \pm 7.0$ & $-0.7 \pm 11.4$ & $1.3 \pm 7.2$ & $5.3 \pm 11.6$ \\
\hline \multicolumn{5}{|l|}{ Stage VI } \\
\hline$\Delta$ SUVR & $-0.02 \pm 0.09$ & $-0.02 \pm 0.13$ & $-0.01 \pm 0.08$ & $0.03 \pm 0.15$ \\
\hline$\%$ & $-1.6 \pm 7.6$ & $-1.7 \pm 12.0$ & $-0.7 \pm 6.9$ & $2.2 \pm 10.5$ \\
\hline
\end{tabular}

cognition worsened showed an increase in SUVR in all cortical areas except the sensorimotor and anterior cingulate cortices, most prominently in the lateral temporal cortices and medial temporal regions (Fig. 3B).

In $62 \mathrm{~A} \beta$-positive individuals, the change in global cognition as determined by the MMSE score correlated with the change in SUVR in the prefrontal, sensorimotor, parietal, occipital, lateral temporal, cingulate, and insula cortices, and the change in language function correlated with the change in diffuse cortical SUVR in the prefrontal, sensorimotor, parietal, occipital, lateral temporal, cingulate, and insular cortices even after correction for multiple comparisons (Supplemental Table 2). These changes still correlated even after adjustment for baseline SUVR in a smaller number of regions (MMSE: prefrontal, sensorimotor, superior parietal, precuneal, and occipital cortices; language: prefrontal, sensorimotor, superior parietal, precuneal, and posterior cingulate cortices). Meanwhile, changes in memory, attention, visuospatial, and frontal/executive function did not correlate with the change in cortical ${ }^{18}$ F-flortaucipir uptake.

\section{Longitudinal Changes in ${ }^{18} \mathrm{~F}$-Flortaucipir Uptake Measured with PERSI Reference Region}

We repeated the same analyses with the white matter-based PERSI reference region (12). In all groups, the areas with a significant increase in ${ }^{18} \mathrm{~F}$-flortaucipir uptake obtained using the PERSI reference region were similar to but wider than those obtained using the cerebellar crus (Supplemental Tables 3 and 4; Supplemental Figs. 2-5). PVE-corrected global cortical 




FIGURE 2. Longitudinal changes in PVE-corrected ${ }^{18} \mathrm{~F}$-flortaucipir SUVR obtained with cerebellar crus as reference region. Regional changes and their statistical significance are shown at top. Horizontal bars in leftward (green) and rightward (red) directions represent $P$ values in log scale with decrease and increase of regional uptake at follow-up, respectively. Vertical blue dotted lines represent cutoff $P$ value of 0.05 (-log $\left.{ }_{10} P=1.3\right)$, and asterisks represent regions that survived correction for multiple comparisons. Surface-based analyses of longitudinal changes are shown at bottom. Mean increases in cortical ${ }^{18} \mathrm{~F}$-flortaucipir SUVR (left) and cortical areas with significantly increased uptake at follow-up compared with those at baseline (right) are displayed on cortical surface. Only vertices that survived correction for multiple comparisons are displayed. For simplicity, negative changes are not displayed.

${ }^{18}$ F-flortaucipir SUVR increased at follow-up in the MCI+ and DEM+ groups by $0.12(6.7 \%)$ and $0.14(7.6 \%)$, respectively (Supplemental Tables 3 and 4). Like the results for the cerebellar crus reference region, both the MCI+ group and the DEM+ group exhibited the most prominently increased uptake in the medial and lateral temporal regions. The greatest increase was in the stage I-II region in the $\mathrm{MCI}+$ group ( $\triangle \mathrm{SUVR}, 0.34[13.3 \%])$ and in the stage III-IV region in the DEM+ group ( $\Delta$ SUVR, 0.23 [11.1\%]). Although the surface-based $\Delta$ SUVR map of the CU+ group showed an increase in diffuse uptake in the basal temporal and lateral 


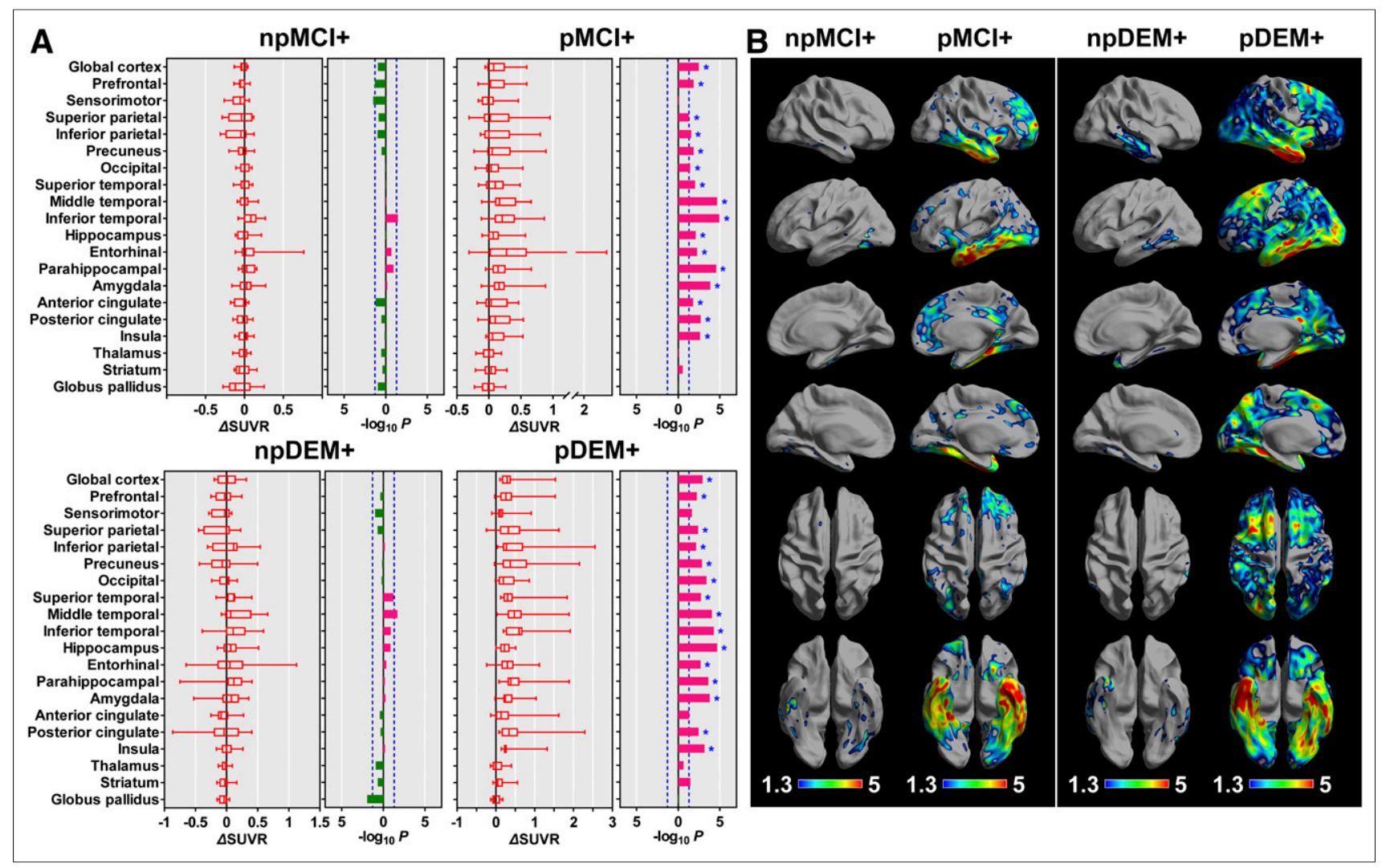

FIGURE 3. Longitudinal changes in PVE-corrected ${ }^{18} \mathrm{~F}$-flortaucipir SUVR obtained with cerebellar crus as reference region in $\mathrm{MCl}$ patients who progressed or did not progress to dementia and in AD patients who showed or did not show progression of cognitive dysfunction. (A) In VOI-based analysis, color bars represent $P$ values in log scale, and horizontal bars in leftward (green) and rightward (red) directions represent $P$ values in log scale with decrease and increase of regional uptake at follow-up, respectively. Vertical blue dotted lines represent cutoff $P$ value of $0.05\left(-\log _{10} P=\right.$ 1.3), and asterisks represent regions that survived correction for multiple comparisons. (B) In surface-based analysis, only vertices that survived correction for multiple comparisons are displayed. For simplicity, negative changes are not displayed. $\mathrm{npMCl}+=\mathrm{MCl}+$ who did not progress to dementia; $\mathrm{pMCl}+=\mathrm{MCl}+$ who progressed to dementia; $\mathrm{npDEM}+=\mathrm{DEM}+$ who did not show progression; $\mathrm{pDEM}+=\mathrm{DEM}+$ who showed progression.

temporal cortices, no region reached statistical significance in this group (Supplemental Fig. 3). Widespread cortical areas exhibited a significant increase in ${ }^{18} \mathrm{~F}$-flortaucipir uptake in the MCI + individuals who progressed to dementia and in DEM+ individuals whose global cognition worsened (Supplemental Fig. 4). The change in global cognition, language, and visuospatial function correlated with the change in ${ }^{18} \mathrm{~F}$-flortaucipir uptake, especially in the prefrontal cortex (Supplemental Table 5). The change in PVE-uncorrected global cortical SUVR $(\Delta$ SUVR in the MCI+ and DEM+ groups, 0.03 [3.0\%]) and the extent of regions with a significant increase in the DEM+ group were much smaller than those after PVE correction (Supplemental Tables 3 and 4; Supplemental Fig. 5).

\section{Longitudinal Changes in ${ }^{18} \mathrm{~F}$-Florbetaben Uptake and Progression of Atrophy}

Only the MCI+ and DEM+ groups showed a significant increase in ${ }^{18} \mathrm{~F}$-florbetaben SUVR (global cortical $\triangle$ SUVR: MCI+, 0.13 [6.0\%]; DEM+, 0.16 [6.7\%]) (Supplemental Fig. 6). The $\mathrm{MCI}+$ group exhibited an increase in widespread cortical areas including the lateral temporal, occipital, insular, parahippocampal, prefrontal, precuneal, posterior cingulate, and superior parietal cortices, and the DEM + group exhibited an increase in the lateral temporal, occipital, parahippocampal, insular, precuneal, inferior parietal, and posterior cingulate cortices and the hippocampus. The CU - group did not show a significant increase in ${ }^{18} \mathrm{~F}$-florbetaben SUVR in any region, and the change in global cortical SUVR was close to zero $(-0.005)$.

Progression of volume atrophy and cortical thinning was most prominent in the DEM+ group in the temporal cortex and diffusely in the frontoparietooccipital cortices (Supplemental Table 6; Supplemental Fig. 7). The MCI+ group showed a significant progression of cortical atrophy in the prefrontal, occipital, lateral temporal, entorhinal, and parahippocampal cortices. The DEM+ group exhibited additional cortical areas (superior and inferior parietal cortices, hippocampus, and amygdala) with progressive volume atrophy. The CU - group showed progressive atrophy in the prefrontal, middle temporal, and parahippocampal cortices, the hippocampus, and the amygdala, whereas only the middle temporal cortex showed progressive atrophy in the $\mathrm{CU}+$ group (Supplemental Fig. 7).

In $62 \mathrm{~A} \beta$-positive individuals, there was a correlation between the change in cortical ${ }^{18} \mathrm{~F}$-flortaucipir and ${ }^{18} \mathrm{~F}$-florbetaben SUVR in the occipital cortex and the hippocampus. The change in cortical ${ }^{18} \mathrm{~F}$-flortaucipir SUVR correlated with the progression of diffuse volume atrophy in the frontotemporoparietal cortices and in the prefrontal, superior parietal, lateral temporal, and entorhinal 
cortices and thalamus. Meanwhile, the change in cortical ${ }^{18} \mathrm{~F}$-florbetaben SUVR weakly correlated with the progression of volume atrophy in the prefrontal, inferior temporal, and parahippocampal cortices, the hippocampus, and the striatum. However, this correlation failed to survive correction for multiple comparisons (Supplemental Fig. 8).

\section{DISCUSSION}

In this study, we identified progressive cortical tau accumulation in prodromal AD and DEM patients. During the 2-y followup, global cortical SUVR after correction for PVE increased in the $\mathrm{MCI}+$ and DEM+ groups by $0.06(2.9 \%)$ and $0.19(8.0 \%)$, respectively. Uptake increased diffusely in the cortical areas, most prominently in the medial and inferior temporal regions in the $\mathrm{MCI}+$ group and in the lateral temporal cortices in the DEM+ group. Meanwhile, global cortical uptake in the $\mathrm{CU}-$ and $\mathrm{CU}+$ groups barely changed during the follow-up period, although there was a small trend toward an increase in the medial temporal areas. Additional tau accumulation was widespread in the association neocortex in $\mathrm{MCI}+$ patients who progressed to dementia and in DEM+ patients with worsening global cognition. A deterioration in global cognition and language function correlated with the progressive tau accumulation in the association neocortex.

NFT pathologic findings first appear in the transentorhinal and entorhinal cortex and then in the neighboring medial and basal temporal cortices. Distant neocortical association areas are involved later, and finally the disease reaches the primary cortices $(20,21)$. Likewise, in vivo tau PET studies on AD patients also showed distinct regional uptake patterns $(3,4,6,7)$ and a regional frequency of tau involvement (2) that closely resemble the sequential pattern of postmortem NFT disease. These findings support the hypothesis that tau spreads hierarchically through anatomically and functionally connected networks $(2,20,22,23)$. Similar to these cross-sectional observations, our longitudinal study showed that the tau accumulation rate in the entorhinal cortex (stages I-II) was greatest in the MCI+ group (0.27 [9.3\%]), followed by DEM+ group $(0.21[6.9 \%])$ and the CU+ group $(0.13$ [6.6\%]). In addition, the tau accumulation rate was greatest in the entorhinal cortex and was lower in regions of higher Braak stage in both the CU+ group and the MCI+ group, whereas it was greatest in the composite stage III-IV region in the DEM+ group. Likewise, the regions showing the most significant increase in uptake in the MCI+ group were more ventral to the regions in the DEM+ group at the VOI and surface level. These findings provide indirect evidence for hierarchical tau spreading from the entorhinal cortex with progression of the disease.

In a large-scale longitudinal ${ }^{18} \mathrm{~F}$-flortaucipir PET study, tau accumulation rates in the entorhinal cortex and amygdala did not differ between the $A \beta$-positive groups with and without cognitive impairment, and continuous tau accumulation even in regions of early Braak stage was suggested (11). Meanwhile, saturation of tau load may be expected by the pathologic changes occurring in regions of early Braak stage. In the Braak original report, tau steadily increased even in the transentorhinal and entorhinal cortices beyond stages I-II. After neuronal death, intraneuronal NFTs are replaced by ghost tangles in the transentorhinal cortex from stage IV, and numerous ghost tangles can be found throughout the medial temporal cortex in stage VI (20). When we consider that ghost tangles can be strongly labeled by ${ }^{18} \mathrm{~F}$ flortaucipir (24), its uptake in the transentorhinal and entorhinal cortex can be expected to increase until advanced stages and to plateau. Although we did not find a negative correlation between baseline uptake and rate of accumulation, the increase in uptake in the entorhinal cortex was greater in the MCI+ group than in the DEM+ group. We suspect that the tau accumulation rate is attenuated in areas below the tau stage at which the most active pathologic process of tau occurs. In addition, there was high variability in regional changes in the MCI+ and DEM+ groups, as seen in the previous longitudinal studies $(11,12)$. Although there might be a bias caused by the image-processing steps, this high variability implies that there may be differences in the region of most active tau accumulation in different patients, as seems likely given that $\mathrm{AD}$ can present with different phenotypes that in turn correspond to the topography of tau uptake.

We also found a relationship between progressive tau accumulation and cognitive decline. MCI+ patients who progressed to dementia and DEM+ patients whose global cognition worsened exhibited progressive diffuse tau accumulation in the temporal and extratemporal association cortices, whereas the nonprogressors showed a slight trend toward an increase in small areas in the basal temporal (MCI+ nonprogressors) and lateral temporal (DEM + nonprogressors) cortices. Likewise, the extent of a change in diffuse uptake in the association cortices correlated with the degree of decline in global cognition and language functions. Therefore, the progression of cognitive dysfunction is related to additional tau accumulation in regions of higher Braak stage. Unlike the language function, which deteriorates later than the memory function (25), the change in uptake did not correlate with the change in memory function. This finding may be due to a floor effect in memory performance such that changes in memory performance were too small to show a correlation with the uptake change.

It is interesting to note that the $\mathrm{CU}-$ group showed a weak agerelated increase in the tau accumulation rate in the entorhinal cortex, a small trend toward an increase in medial temporal uptake on the VOI-based analysis, and an additional increase in portions of the basal temporal regions on surface-based analysis. This trend was better visualized when the PERSI reference region was used. These findings suggest that tau accumulation in the medial temporal regions of elderly individuals - the primary age-related tauopathy (26) — may not be stationary but may steadily increase within or even beyond the medial temporal regions. However, it still remains unclear whether primary age-related tauopathy is a continuum of $\mathrm{AD}$. To support this hypothesis, we need to observe a progression of tau accumulation beyond the medial temporal regions after the conversion of $A \beta$ positivity in elderly individuals suspected of having primary age-related tauopathy in a long-term follow-up study.

According to the hypothetical model of the dynamic biomarkers for $\mathrm{AD}$ (27), the surge of $\mathrm{A} \beta$ accumulation precedes that of tau accumulation, and then the neurodegeneration follows. This model was replicated in our cross-sectional tau and amyloid PET studies $(2,25)$. In the present longitudinal study, the increase in ${ }^{18} \mathrm{~F}$-florbetaben uptake in the MCI+ group was as high as the increase in the DEM+ group, whereas the increase in ${ }^{18} \mathrm{~F}$-flortaucipir uptake in the DEM + group was almost 3 times greater than that in the MCI+ group. Progressive tau accumulation occurred in widespread cortical areas and was attenuated in the entorhinal 
cortex in the DEM+ group. Unlike this pattern, the progression of volume atrophy appeared prominently in the lateral temporal cortex and in a part of the medial temporal regions in the MCI+ group and still more prominently in the medial temporal regions in the DEM + group. These results suggest that $A \beta$ accumulation may accelerate in the MCI stage of AD and then may be followed by acceleration of tau accumulation in the later dementia stage of AD. Progression of volume atrophy may be sustained even after the tau accumulation plateaus. These patterns of longitudinal change in the imaging biomarkers for $\mathrm{A} \beta$, tau, and neurodegeneration strongly support the hypothetical model.

Compared with the previous longitudinal study using the PERSI reference region (PVE-uncorrected and weighted $\triangle$ SUVR in the A $\beta$-positive AD patients, 0.05/y) (12), PVE-uncorrected $\Delta$ SUVR obtained with the PERSI reference region in the present study was lower than previously reported (global cortical $\triangle$ SUVR in the $\mathrm{DEM}+$ group, 0.015/y). Although Southekal et al. (12) reported a change in SUVR that was more weighted on the temporal cortex and thereby their $\triangle$ SUVR should be greater than our global cortical $\Delta$ SUVR, our annual change in SUVR in the composite stage I-IV region was still lower $(\triangle$ SUVR in the DEM+ group, $0.028 / y$ ) than theirs. Likewise, compared with a previous longitudinal ${ }^{18} \mathrm{~F}$-flortaucipir PET study using the cerebellar crus as a reference tissue by Jack et al. (11), the annual increase in global cortical ${ }^{18} \mathrm{~F}$-flortaucipir SUVR uncorrected for PVE was smaller in our 55 patients with cognitive impairment (Jack et al., $0.040 / y$, vs. ours, $0.026 / y$ ). We suspect that this discrepancy might be attributable to differences in severity in patients, image-processing steps, and VOIs for measuring regional uptake. However, PVE correction greatly affected the results in our study, and thereby, the annual increase was almost similar after correction for PVE (Jack et al., 0.053/y, vs. ours, 0.060/y). Because of the large effect of progressive atrophy in the DEM+ group, the change was less than half that obtained after PVE correction, and only the lateral temporal cortices showed a significant increase in uptake in the DEM + group without PVE correction. We also calculated the change in uptake with data corrected for PVE by the Meltzer method (28) and found almost similar results (global cortical $\triangle$ SUVR in 55 CI+ individuals, 0.059/y) (Supplemental Table 7). However, there still remains concern about overcorrection of cortical uptake by PVE correction and thereby overestimation of the change.

In the DEM + group, $\triangle$ SUVR and the SUVR obtained by the PERSI reference region were generally lower than those obtained for the cerebellar crus reference, possibly because of incomplete elimination of voxels with high target binding from the PERSI reference region. Although ${ }^{18} \mathrm{~F}$-flortaucipir PET exhibits no prominent white matter uptake compared with the ${ }^{18} \mathrm{~F}-\mathrm{THK}$ series tau tracers, the ${ }^{18} \mathrm{~F}$-flortaucipir SUV in the global white matter of the $\mathrm{CU}-$ group was about $8 \%$ greater than in the global cortex in our study. Nevertheless, the PERSI reference region provided a greater effect size for groupwise longitudinal change in uptake than the cerebellar crus in the PVE-uncorrected data (Cohen d for global cortical $\triangle$ SUVR in MCI+ group: 0.741 for PERSI vs. 0.294 for crus; Cohen d in DEM+ group: 0.467 for PERSI vs. 0.433 for crus) and in the PVE-corrected data (Cohen $\mathrm{d}$ for global cortical $\triangle \mathrm{SUVR}$ in MCI+ group: 0.927 for PERSI vs. 0.418 for crus; Cohen d in DEM+ group: 0.958 for PERSI vs. 0.566 for crus). Additionally, small increases in uptake in the medial temporal region in the $\mathrm{CU}-$ group were visualized using the PERSI reference region. Therefore, we suspect that the PERSI reference region is more sensitive for showing changes in uptake in longitudinal ${ }^{18} \mathrm{~F}$-flortaucipir PET studies than is the classic cerebellar crus reference.

Although a greater change in SUVR in the MCI+ and DEM+ groups than the variability of SUVR in a test-retest study of ${ }^{18} \mathrm{~F}$ flortaucipir PET suggests a true increase in tau burden (29), instability and an overestimation bias of ${ }^{18} \mathrm{~F}$-flortaucipir SUVR might have caused some bias in estimating the change in SUVR in our longitudinal study (30).

According to the previous cross-sectional tau PET studies showing a greater tau accumulation in early-onset $\operatorname{AD}(5,8,9)$, we may expect a negative correlation between baseline age and change in SUVR. However, there was only a small trend toward a negative correlation between 2 variables in this longitudinal study. This negative result may be attributable to the small number of younger AD patients included in our study (6 patients with age $<$ $65 \mathrm{y}$ among $62 \mathrm{~A} \beta$-positive participants) and the fact that patients with an atypical clinical presentation (who tend to be younger) were not included in this study. Younger AD patients are likely to show rapid progression $(31,32)$ and were unable to undergo the entire assessment because of markedly progressed cognitive dysfunction at follow-up. This problem limited the extent of areas with significantly increased uptake in the DEM + group at follow-up.

\section{CONCLUSION}

Our study revealed progressive tau accumulation predominantly in the medial and basal temporal cortices in prodromal AD and in the lateral temporal cortices in DEM patients. These progressive tau accumulation patterns support the Braak hypothetical model of pathologic tau propagation. In addition, the propagation of tau to regions of higher Braak stage is associated with worsening of cognitive dysfunction in AD. We confirmed that ${ }^{18} \mathrm{~F}$-flortaucipir PET is an imaging biomarker for monitoring the progression of $\mathrm{AD}$.

\section{DISCLOSURE}

This research was supported by the Basic Science Research Program through the National Research Foundation of Korea (NRF) funded by the Ministry of Science, ICT, and Future Planning (NRF-2017R1A2B2006694), which is funded by the Ministry of Education (NRF-2018R1D1A1B07049386). This research was also supported by a grant from the Korea Health Technology R\&D Project through the Korea Health Industry Development Institute (KHIDI), which is funded by the Ministry of Health and Welfare, Republic of Korea (grant HI18C1159). Support was also received through a faculty research grant from Yonsei University College of Medicine (62018-0068). Clifford Jack, Jr., consults for Lily and serves on an independent data monitoring board for Roche but receives no personal compensation from any commercial entity. He receives research support from the NIH and the Alexander Family Alzheimer's Disease Research Professorship of the Mayo Clinic. No other potential conflict of interest relevant to this article was reported. 


\section{ACKNOWLEDGMENTS}

We express our special appreciation to Tae Ho Song and Won Taek Lee (PET technologists), who managed all the PET scans with enthusiasm.

\section{KEY POINTS}

QUESTION: Does longitudinal change in cortical tau burden measured by ${ }^{18} \mathrm{~F}$-flortaucipir PET reflect progression of $A D$ ?

PERTINENT FINDINGS: Our 2-y longitudinal tau PET study showed a $2.9 \%$ increase in cortical ${ }^{18} \mathrm{~F}$-flortaucipir SUVR in prodromal $A D$ and an $8 \%$ increase in DEM patients. Deterioration of global cognition and thereby conversion to a more advanced clinical $A D$ status were associated with the progression of diffuse tau accumulation in the association neocortex.

IMPLICATIONS FOR PATIENT CARE: ${ }^{18} \mathrm{~F}$-flortaucipir PET is an imaging biomarker for monitoring the progression of $A D$.

\section{REFERENCES}

1. Saint-Aubert L, Lemoine L, Chiotis K, Leuzy A, Rodriguez-Vieitez E, Nordberg A. Tau PET imaging: present and future directions. Mol Neurodegener. 2017;12:19.

2. Cho H, Choi JY, Hwang MS, et al. In vivo cortical spreading pattern of tau and amyloid in the Alzheimer disease spectrum. Ann Neurol. 2016;80:247-258.

3. Cho H, Choi JY, Hwang MS, et al. Tau PET in Alzheimer disease and mild cognitive impairment. Neurology. 2016;87:375-383.

4. Johnson KA, Schultz A, Betensky RA, et al. Tau positron emission tomographic imaging in aging and early Alzheimer disease. Ann Neurol. 2016;79:110-119.

5. Ossenkoppele R, Schonhaut DR, Scholl M, et al. Tau PET patterns mirror clinical and neuroanatomical variability in Alzheimer's disease. Brain. 2016;139: 1551-1567.

6. Schöll M, Lockhart SN, Schonhaut DR, et al. PET imaging of tau deposition in the aging human brain. Neuron. 2016;89:971-982.

7. Schwarz AJ, Yu P, Miller BB, et al. Regional profiles of the candidate tau PET ligand ${ }^{18} \mathrm{~F}-\mathrm{AV}-1451$ recapitulate key features of Braak histopathological stages. Brain. 2016;139:1539-1550.

8. Cho H, Choi JY, Lee SH, et al. Excessive tau accumulation in the parietooccipital cortex characterizes early-onset Alzheimer's disease. Neurobiol Aging. 2017;53:103-111.

9. Lowe VJ, Wiste HJ, Senjem ML, et al. Widespread brain tau and its association with ageing, Braak stage and Alzheimer's dementia. Brain. 2018;141:271-287.

10. Phillips JS, Das SR, McMillan CT, et al. Tau PET imaging predicts cognition in atypical variants of Alzheimer's disease. Hum Brain Mapp. 2018;39:691-708.

11. Jack CR Jr, Wiste HJ, Schwarz CG, et al. Longitudinal tau PET in ageing and Alzheimer's disease. Brain. 2018;141:1517-1528.

12. Southekal S, Devous MD Sr, Kennedy I, et al. Flortaucipir F 18 quantitation using parametric estimation of reference signal intensity. J Nucl Med. 2018;59: 944-951.
13. Sabri O, Sabbagh MN, Seibyl J, et al. Florbetaben PET imaging to detect amyloid beta plaques in Alzheimer's disease: phase 3 study. Alzheimers Dement. 2015;11: 964-974.

14. Villemagne VL, Ong K, Mulligan RS, et al. Amyloid imaging with ${ }^{18} \mathrm{~F}$-florbetaben in Alzheimer disease and other dementias. J Nucl Med. 2011;52:1210-1217.

15. McKhann GM, Knopman DS, Chertkow H, et al. The diagnosis of dementia due to Alzheimer's disease: recommendations from the National Institute on AgingAlzheimer's Association workgroups on diagnostic guidelines for Alzheimer's disease. Alzheimers Dement. 2011;7:263-269.

16. Albert MS, DeKosky ST, Dickson D, et al. The diagnosis of mild cognitive impairment due to Alzheimer's disease: recommendations from the National Institute on Aging-Alzheimer's Association workgroups on diagnostic guidelines for Alzheimer's disease. Alzheimers Dement. 2011;7:270-279.

17. Kang Y, Na DL. Seoul Neuropsychological Screening Battery (SNSB). Incheon, South Korea: Human Brain Research \& Consulting Co.; 2003.

18. Thomas BA, Erlandsson K, Modat M, et al. The importance of appropriate partial volume correction for PET quantification in Alzheimer's disease. Eur J Nucl Med Mol Imaging. 2011;38:1104-1119.

19. Benjamini Y, Hochberg Y. Controlling the false discovery rate: a practical and powerful approach to multiple testing. J R Stat Soc B. 1995;57:289-300.

20. Braak H, Braak E. Neuropathological stageing of Alzheimer-related changes. Acta Neuropathol (Berl). 1991;82:239-259.

21. Braak H, Alafuzoff I, Arzberger T, Kretzschmar H, Del Tredici K. Staging of Alzheimer disease-associated neurofibrillary pathology using paraffin sections and immunocytochemistry. Acta Neuropathol (Berl). 2006;112:389-404.

22. de Calignon A, Polydoro M, Suarez-Calvet M, et al. Propagation of tau pathology in a model of early Alzheimer's disease. Neuron. 2012;73:685-697.

23. Ahmed Z, Cooper J, Murray TK, et al. A novel in vivo model of tau propagation with rapid and progressive neurofibrillary tangle pathology: the pattern of spread is determined by connectivity, not proximity. Acta Neuropathol (Berl). 2014;127:667-683.

24. Ono M, Sahara N, Kumata K, et al. Distinct binding of PET ligands PBB3 and AV-1451 to tau fibril strains in neurodegenerative tauopathies. Brain. 2017; 140:764-780.

25. Cho H, Lee HS, Choi JY, et al. Predicted sequence of cortical tau and amyloid-beta deposition in Alzheimer disease spectrum. Neurobiol Aging. 2018;68:76-84.

26. Crary JF, Trojanowski JQ, Schneider JA, et al. Primary age-related tauopathy (PART): a common pathology associated with human aging. Acta Neuropathol (Berl). 2014;128:755-766.

27. Jack CR Jr, Knopman DS, Jagust WJ, et al. Tracking pathophysiological processes in Alzheimer's disease: an updated hypothetical model of dynamic biomarkers. Lancet Neurol. 2013;12:207-216.

28. Meltzer CC, Leal JP, Mayberg HS, Wagner HN Jr, Frost JJ. Correction of PET data for partial volume effects in human cerebral cortex by MR imaging. J Comput Assist Tomogr. 1990;14:561-570.

29. Devous MD Sr, Joshi AD, Navitsky M, et al. Test-retest reproducibility for the tau PET imaging agent flortaucipir F 18. J Nucl Med. 2018;59:937-943.

30. Wooten DW, Guehl NJ, Verwer EE, et al. Pharmacokinetic evaluation of the tau PET radiotracer ${ }^{18} \mathrm{~F}-\mathrm{T} 807\left({ }^{18} \mathrm{~F}-\mathrm{AV}-1451\right)$ in human subjects. J Nucl Med. 2017; 58:484-491.

31. Heyman A, Wilkinson WE, Hurwitz BJ, et al. Early-onset Alzheimer's disease: clinical predictors of institutionalization and death. Neurology. 1987;37:980-984.

32. Koss E, Edland S, Fillenbaum G, et al. Clinical and neuropsychological differences between patients with earlier and later onset of Alzheimer's disease: a CERAD analysis, part XII. Neurology. 1996;46:136-141. 